\title{
BOUNDED SETS IN $(F)$-SPACES
}

\author{
JEAN DIEUDONNÉ
}

1. In the theory of $(F)$-spaces, ${ }^{1}$ the following two questions arise:

(A) Let $E$ be a metrizable locally convex space; is it true that if every bounded set in $E$ is separable, the space $E$ is separable?

(B) Let $E$ be a metrizable locally convex space, $\bar{E}$ its completion; is it true that every bounded set in $\bar{E}$ is contained in the closure of a bounded set of $E$ ?

The purpose of this note is to prove that, if we assume the continuum hypothesis, the answer to both questions is negative.

2. We begin by proving a lemma which is substantially well known (see e.g. [2]; the continuum hypothesis is used at this place).

Lemma. Let us well-order the set $\boldsymbol{N}^{N}$ of all sequences of positive integers in a transfinite sequence $\left(f_{\lambda}\right)$ of type $\omega_{1}$; then there is a subset $A$ of ordinals, having the power of the continuum, such that if $\alpha, \beta$ are in $A$ and $\alpha<\beta, f_{\alpha}=o\left(f_{\beta}\right)$, and that, for every ordinal $\lambda<\omega_{1}$, there is an $\alpha>\lambda$ in $A$ such that $f_{\lambda}=o\left(f_{\alpha}\right)$. We can moreover suppose that $f_{\alpha}$ is a strictly increasing sequence for every $\alpha \in A$.

Suppose we have defined all elements of $A$ which are $<\alpha$; the ordinals $<\alpha$ constituting a denumerable set, there is, by $\mathrm{Du}$ BoisReymond's theorem, a strictly increasing sequence $f_{\lambda}$ with $f_{\mu}=o\left(f_{\lambda}\right)$ for every $\mu<\alpha$; ordinals $\lambda$ having that property are obviously $\geqq \alpha$, and we take as the first element of $A$ which is $\geqq \alpha$, the smallest of these ordinals $\lambda$. It is clear that $A$ has the power of the continuum.

As a corollary, it follows that if $B$ is a subset of $A$ such that there exists an ordinal $\alpha \in A$ such that $f_{\beta}=O\left(f_{\alpha}\right)$ for every $\beta \in B$, then $B$ is denumerable, for the indices of $B$ are necessarily $<\alpha$.

3 . In the product space $R^{N}$ (space of all sequences of real numbers, with the topology of pointwise convergence), let us consider the set $S$ of all elements of the form $g_{n}\left(f_{\alpha}+m\right)$, where $\alpha \in A, m$ is an arbitrary integer (positive or negative), and $g_{n}$ (for every integer $n \geqq 0$ ) is the sequence $\left(\xi_{n k}\right)_{k \geqq 0}$ where $\xi_{n k}=0$ for $k<n, \xi_{n k}=1$ for $k \geqq n$. In other words, $g_{n}\left(f_{\alpha}+m\right)$ is the sequence $\left(\xi_{k}\right)$ such that $\xi_{k}=0$ for $k<n, \xi_{k}=f_{\alpha}(k)+m$ for $k \geqq n$. On the other hand, let $F$ be the Banach space of all real bounded functions $\left(x_{\alpha}\right)_{\alpha \in A}$, with the usual norm $\sup _{\alpha \in A}\left|x_{\alpha}\right|$. For each $\alpha \in A$, let $e_{\alpha}$ be the element of $F$, whose coordinates are all 0 , ex-

Received by the editors October 23, 1954.

${ }^{1}$ We use the terminology of [1] and [3]. 
cept that of index $\alpha$, which is equal to 1 ; we observe that $\left\|e_{\alpha}\right\|=1$ and that the distance of $e_{\alpha}$ to the closed subspace of $F$ generated by the $e_{\beta}$ of index $\beta \neq \alpha$ is 1 .

In the product space $F \times R^{N}$, consider now the set of all elements $\left(e_{\alpha}, g_{n}\left(f_{\alpha}+m\right)\right)$, where $\alpha$ ranges over $A, n$ over all integers $\geqq 0, m$ over all positive or negative integers; and let $E$ be the subspace of $F \times R^{N}$ generated by all finite linear combinations of these elements. It is clear that $E$ is locally convex and metrizable; on the other hand, the projection of $E$ on $F$ is not separable, hence $E$ is not separable.

Let now $H$ be a bounded set in $E$; its projection $L$ on $R^{N}$ is then bounded, which means that there exists an ordinal $\mu<\omega_{1}$ such that all elements of $L$ are $O\left(f_{\mu}\right)$. Now it is clear that every element $z=(x, y)$ of $E$ can be written in one and only one way

$$
z=\sum_{i} \lambda_{i}\left(e_{\alpha_{i}}, f_{\alpha_{i}}\right)+(0, u)
$$

where $u$ belongs to the set $U$ of all ultimately constant sequences (i.e., constant after a certain term), the $\alpha_{i}$ are distinct ordinals of $A$, finite in number (eventually equal to 0 ), and the $\lambda_{i}$ are $\neq 0$. If $z \notin U$, we have $y \sim \lambda_{k} f_{\alpha_{k}}$, where $\alpha_{k}$ is the largest of the $\alpha_{i}$; hence in all elements $z \in H$ which are not in $U$, the $\alpha_{i}$ are bounded by a common $\beta \in A$ (from the lemma in $\$ 2$ ), and therefore constitute a denumerable set. As $U$ is obviously a separable subset of $R^{N}$, we have proved that each bounded subset $H$ of $E$ is separable, thus getting a negative answer to problem (A).

4. Let now $\bar{E}$ be the completion of $E$, which we can identify with the closure of $E$ in the product space $F \times R^{N}$. Let $B$ be the bounded subset of $\bar{E}$ consisting of all elements $(x, y)$ of that space such that $\|x\| \leqq 2$ and $|y(n)| \leqq 1$ for every $n$. We are going to prove that $B$ is not separable, thus answering problem (B) negatively.

Let $\alpha$ be an arbitrary element of $A$; as $A$ is nondenumerable, there is an infinite sequence $\left(\alpha_{k}\right)$ of elements of $A$ such that $\alpha_{0}=\alpha, \alpha_{k}<\alpha_{k+1}$ hence $f_{\alpha_{k}}=o\left(f_{\alpha_{k+1}}\right)$. We are going to determine a convergent sequence $\left(h_{k}\right)$ in the space $R^{N}$ in the following way. Let the integer $m_{0}$ be determined such that $\left|f_{\alpha_{0}}(0)+m_{0}\right| \leqq 1$, and take $h_{0}=g_{0}\left(f_{\alpha_{0}}+m_{0}\right)$. Let $n_{1}$ be the first integer for which $\left|h_{0}(n)\right|>1$; we determine the integer $m_{1}$ such that $f_{\alpha_{1}}\left(n_{1}\right)+m_{1} \geqq 2\left|h_{0}\left(n_{1}\right)\right|$, and finally, we take $h_{1}=h_{0}+\lambda_{1} g_{n_{1}}$ - $\left(f_{\alpha_{1}}+m_{1}\right), \lambda_{1}$ being a real number determined by the equation $h_{1}\left(n_{1}\right)$ $=0$; it is clear that $\left|\lambda_{1}\right| \leqq 1 / 2$. Let $n_{2}$ be the first integer for which $\left|h_{1}(n)\right|>1$; we take $m_{2}$ such that $f_{\alpha_{2}}\left(n_{2}\right)+m_{2} \geqq 2^{2}\left|h_{1}\left(n_{2}\right)\right|$, and then we form $h_{2}=h_{1}+\lambda_{2} g_{n_{2}}\left(f_{\alpha_{2}}+m_{2}\right), \lambda_{2}$ being determined by the equation $h_{2}\left(n_{2}\right)=0$; we have $\left|\lambda_{2}\right| \leqq 1 / 2^{2}$. The induction proceeds in an obvious 
fashion, and, due to the choice of the $\alpha_{k}$, is never stopped. Moreover, $h_{k}(n)$ has, for each $n$, a fixed value from a certain $k$ on, hence the sequence $\left(h_{k}\right)$ converges in $R^{N}$ to an element $y_{\alpha}$ such that $\left|y_{\alpha}(n)\right| \leqq 1$ for each $n$. But $h_{k}$ is the projection of the element

$$
\left(e_{\alpha_{0}}+\lambda_{1} e_{\alpha_{1}}+\cdots+\lambda_{k} e_{\alpha_{k}}, h_{k}\right)
$$

of $E$, and the sequence of these elements obviously converges to an element $\left(t_{\alpha}, y_{\alpha}\right)$ of $\bar{E}$ which belongs to $B$. To each $\alpha \in A$ we have thus attached an element $z_{\alpha}=\left(t_{\alpha}, y_{\alpha}\right)$ of $B$; moreover the construction is such that, if $\alpha, \beta$ are two indices in $A$ such that $\alpha<\beta$, we have $\left\|t_{\alpha}-t_{\beta}\right\| \geqq 1$. The projection of $B$ on $F$ is thus a nonseparable space, and so therefore is $B$ itself.

5. It would be interesting to give negative answers to problems (A) and (B) without using the continuum hypothesis. On the other hand, if in the statement of problem (A), we require that $E$ be complete (in other words, an $(F)$-space), we obtain a modified problem $\left(\mathrm{A}^{\prime}\right)$ which (even with the continuum hypothesis) remains still unsolved.

\section{BIBLIOGRAPHY}

1. N. Bourbaki, Espaces vectoriels topologiques, Chap. I-II, Actualités Scientifiques et Industrielles, no. 1189, Paris, Hermann, 1953.

2. G. Choquet, Question 0007, Intermédiaire des Recherches Mathématiques vol. 1 (1945) p. 4.

3. J. Dieudonné, Recent developments in the theory of locally convex vector spaces, Bull. Amer. Math. Soc. vol. 59 (1953) pp. 495-512.

NORTHWESTERN UNIVERSITY 